\title{
WAVEGUIDE AND LOOP COUPLING TO FAST MHD TOROIDAL EIGENMODES
}

BY

F, J, PAOLONI

\section{PLASMA PHYSICS LABORATORY}
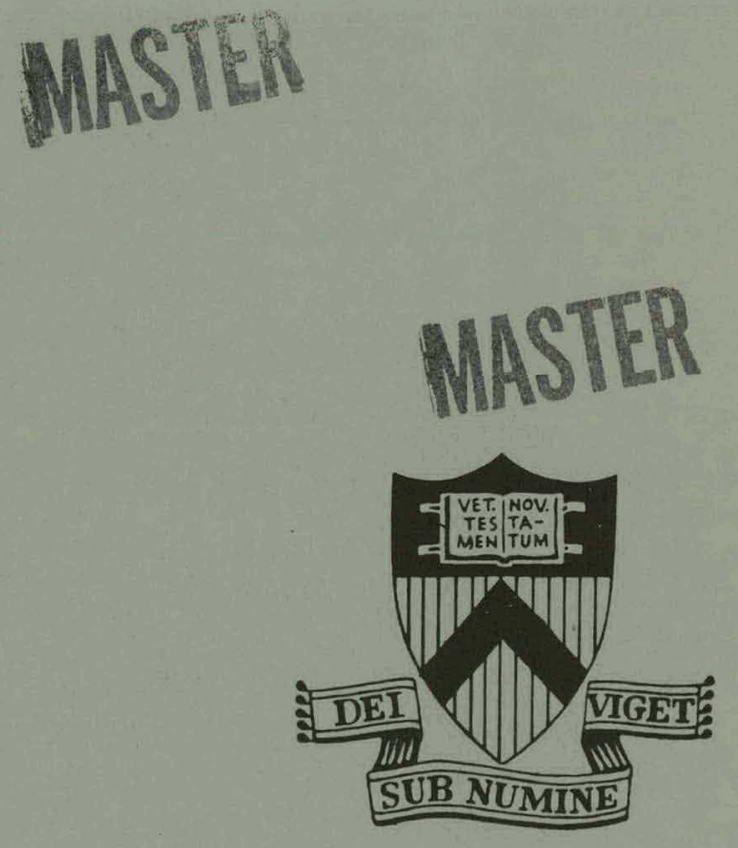

\section{PRINCETON \\ UNIVERSITY PRINCETON, NEW JERSEY}

This work was supported by U. S. Energy Research and Development Administration Contract E(11-1)-3073. Reproduction, translation, publication, use and disposal, in whole or in part, by or for the United States Government is permitted. 


\section{DISCLAIMER}

This report was prepared as an account of work sponsored by an agency of the United States Government. Neither the United States Government nor any agency Thereof, nor any of their employees, makes any warranty, express or implied, or assumes any legal liability or responsibility for the accuracy, completeness, or usefulness of any information, apparatus, product, or process disclosed, or represents that its use would not infringe privately owned rights. Reference herein to any specific commercial product, process, or service by trade name, trademark, manufacturer, or otherwise does not necessarily constitute or imply its endorsement, recommendation, or favoring by the United States Government or any agency thereof. The views and opinions of authors expressed herein do not necessarily state or reflect those of the United States Government or any agency thereof. 


\section{DISCLAIMER}

Portions of this document may be illegible in electronic image products. Images are produced from the best available original document. 


\section{NOTICE}

This report was prepared as an account of work sponsored by the United States Government. Neither the United States nor the United States Energy Research and Development Administration, nor any of their employees, nor any of their contractors, subcontractors, or their employees, makes any warranty, express or implied, or assumes any legal liability or responsibility for the accuracy, completeness or usefulness of any information, apparatus, product or process disclosed, or represents that its use would not infringe privately owned rights.

Printed in the United States of America.

Available from

National Technical Information Service

U. S. Department of Commerce 5285 Port Royal Road

Springfield, Virginia 22151

Price: Printed Copy $\$$ * ; Microfiche $\$ 1.45$

NTIS

*Pages

$$
1-50
$$

$51-150$

$151-325$

$326-500$

$501-1 \cap 00$
Selling Price

$\$ 4.00$

5.45

7.60

10.60

13.60 
Waveguide and Loop Coupling to Fast

MHD Toroidal Eigenmodes

F. J. Paoloni

Plasma Physics Laboratory, Princeton University

Princeton, New Jersey 08540

\section{ABSTRACT}

Heating of plasmas by wave techniques requires an effective method of coupling RF energy to the plasma. In cavities the presence of weakly damped eigenmodes will enhance the loading of antennas when the wave frequency equals an eigenmode frequency: This report considers two methods of coupling to fast MHD eigenmodes in a toroidal cavity: one is by a waveguide mounted perpendicular to the vacuum vessel wall; and the other by a loop placed within the cavity. The effective loading impedance is obtained by equating the input power to the rate at which energy is dissipated within the cavity. The results indicate that the loading is strongly determined by the $Q$ of the cavity and the distance from the antenna to the plasma surface. In PLT, efficicnt coupling to longer wavelength eigenmodes at $60 \mathrm{MHz}$ may be achieved by either a loop or by a loaded waveguide. The guide offers some advantages over an unshielded loop since the aperture fields contain no harmful electric component along the toroidal magnetic field, and no antenna structure is required within the vacuum vessel. The report includes a description of a $35 \mathrm{~cm}$ wide waveguide suitable for PLT which is loaded by titanium dioxide or pure water and sealed from the vacuum vessel by a titanium dioxide slab. The ceramic is protected from the plasma by a metal grid in the form of a Faraday shield. 
INTRODUCTION

Damping of the fast MHD wave in a hot fully ionized plasma can be an effective method of particle heating. Efficiencies of about 20\% were observed by Hooke (1972), (1973), and Adam et al (1974) in the ST Tokamak at Princeton when the wave underwent damping at the second harmonic of the ion cyclotron frequency. In larger machines higher efficiencies are expected. When the waves are heavily damped, little wave energy returns to the antenna for each transit of the machine. Consequently, the situation for fast waves can be considered to be an antenna radiating into a long cylindrical plasma. Some detailed calculations for the loading resistance of a reverse turn (Stix) coil positioned around the plasma have been given by Stix (1962) and Hosea and Sinclair (1970), and calculations for a half turn loop at the plasma edge have been described by Hosea and Hooke (1973). When the wave damping is small, however, appreciable wave energy will return to the antenna at each transit of the torus. By properly selecting the phase velocity of the wave, the loading of the antenna can be increased significantly above the radiation resistance. Such a resonant cavity effect was observed by Adam et al (1974), where the loading resistance of the half lonp increased several orders of magnitude above the radiation resistance. Calculations of the loading of loops in the presence of toroidal eigenmodes have been reported by Paoloni (1975 a).

In large toroidal machines, like PLT, the port is of sufficient size to consider the possibility of coupling to 
fast toroidal eigenmodes by a waveguide mounted perpendicular to the plasma chamber. The antenna electric field is in the poloidal direction and has no toroidal component which can cause harmful particle acceleration along magnetic field lines. The scheme also eliminates the need for an antenna structure, such as a loop, to be placed within the vacuum chamber.

This report describes a method of calculating the loading impedances of waveguides and loops which are coupling to fast MHD eigenmodes in a toroidal cavity. Certain approximations are used to keep the equations in an analytical form, but as indicated in the text, the method can be applied to more complete calculations involving a more detailed plasma model.

\section{WAVEGUIDE COUPLING MODEL}

We wish to consider the coupling of a waveguide of dimensions ( $b \mathrm{x} d$ ) to a resonant cavity through a $\mathrm{H}$ plane aperture of dimensions ( $\left.b x d^{\prime}\right)$ as shown in Fig. I. This can be achieved using a variational technique [Harrington (1961)] where, as a first step, the fields in. the aperture are assumed. By conserving the complex poynting vector calculated at the aperture from both the waveguide side and the cavity side, an expression for the effectlve aperlufe impedanes is obtaincd.

1. Aperture Complex Poynting Vector in the Waveguide

We begin by assuming that the (time harmonic) transverse aperture fields are given by

$$
\begin{array}{rlrl}
{\left[E_{y}\right]_{z=0}} & =E_{0} \sin \frac{\pi x}{d^{\prime}} & x<d^{\prime} \\
& =U & x>d^{\prime}
\end{array}
$$

and $[E x]_{z=0}=0$ 
where the aperture lies in the $\mathrm{z}=0$ plane and the $\mathbf{x} \& \mathbf{y}$ axis lie along the sides of the waveguide.

The aperture fields can now be written as a superposition of $T E_{\text {po }}$ waves in the waveguide such that

$$
\left[E_{y}\right]_{z=0}=E_{0} \sum_{p=1}^{\infty} A_{p o} \sin \frac{p \pi x}{d}
$$

where

$$
{ }_{p o}=\frac{2 d^{\prime}}{\pi d^{\prime}} \frac{\sin \left(p \pi \frac{d^{\prime}}{d}\right)}{\left[1-\left(p \frac{d^{\prime}}{d}\right)^{2}\right]}
$$

Suppose that the waveguide width $d$ is such that all modes other than the $\mathrm{TE}_{10}$ mode are cut-off. The fields in the waveguide (which is to the left of the $z=0$ plane) are this

$$
\begin{gathered}
E_{y}(z<0)=\frac{\left(e^{-i \beta z}+\Gamma e^{i \beta z}\right)}{(1+\Gamma)} E_{0}^{A_{10}} \sin \frac{\pi x}{d} \\
\quad+E_{0} \sum_{p=2}^{\infty} A_{p o} \sin \frac{p \pi x}{d} e^{a} p o z \\
-H_{x}(z<0)=\frac{\left(e^{-i \beta z}-\Gamma e^{i \beta z}\right)}{z_{10}(1+\Gamma)} E_{0} A_{10} \sin \frac{\pi x}{d} \\
-E_{0} \sum_{p=2}^{\infty} \frac{A_{p o}}{z_{p o}} \sin \frac{p \pi x}{d} e^{a_{p o} z}
\end{gathered}
$$


where $\Gamma$ is the complex reflection coefficient for the $\mathrm{TE}_{10}$ mode,

$Z_{\text {po }}$ is the characteristic waveguide impedance for $T E_{\text {po }}$ modes,

$B$ is the wave number for the $\mathrm{TE}_{10}$ mode,

and $a_{p o}$ is the cut-off mode attenuation constant, $p>2$.

In the aperture plane the electric field is real and the complex power averaged across the aperture area can thus be written

$$
\mathrm{P}_{\mathrm{W}}^{*}=\iint_{\text {aperture }}[\underset{\sim}{E} \times \underset{\sim}{\mathrm{H}}]_{\mathrm{z}=0} \cdot \underset{\sim}{\mathrm{d} s} .
$$

Completing the integration using the orthogonality relations between eigenfunctions for the waveguide, we obtain

$$
\mathrm{P}_{\mathrm{W}}^{*}=\frac{b d}{2} E_{0}^{2}\left(\frac{1}{z_{a p}} A_{10}^{2}-\frac{1}{2} \sum_{p=2}^{\infty} \frac{A_{p o}^{2}}{Z_{p o}}\right) .
$$

where the effective aperture impedance is defined by

$$
z_{a p}=z_{10}\left(\frac{I+\Gamma}{1-\Gamma}\right)
$$




$$
\begin{aligned}
& \text { and } \quad z_{10}=n\left[1-\left(E_{10} / E\right)^{2}\right]^{-1 / 2} \\
& z_{p o}=i n\left[\left(\frac{p \lambda_{o}}{2 d}\right)^{2}-1\right]-1 / 2 \quad p \geq 2 \\
& n=\sqrt{\frac{\mu}{\varepsilon}}=\text { characteristic impedance of waveguide medium. } \\
& \mathrm{f}_{10}=1 /(2 \mathrm{~d} \sqrt{\mu \varepsilon})=\text { cut-off frequency of } \mathrm{TE}_{10} \text { mode. } \\
& f \text { is the operating frequency. } \\
& \lambda_{0}=1 /(\sqrt{\mu \varepsilon})=\text { wavelength in the waveguide medium. }
\end{aligned}
$$

\section{Cavity Excitation}

We now consider the excitation of the cavity by the aperture fields. For this purpose, it is convenient to separate the waveguide from the cavity by introducing a "conceptual" magnetic source term as demonstrated in Fig. 2. In this model the aperture fed cavity thus becomes a completely enclosed cavity driven by a surface magnetization

$$
\underset{\sim S}{M}=\underset{\sim}{\mathrm{M}} \times \underset{\sim a p}{\mathrm{E}} \delta(z)
$$

where $\underset{\sim}{\hat{n}}$ is the unit outward normal to the cavity and $\underset{\sim}{\mathrm{E}} \mathrm{ap}$ is the aperture electric field which is non-zero only in the cross-section of the aperture.

Maxwell's equations including the magnetic source term are

$$
\begin{aligned}
& -\nabla \times \underset{\sim}{E}=i \omega \mu_{0} \underset{\sim}{\mathrm{H}}+\underset{\sim}{\mathrm{M}} \\
& \nabla \times \underset{\sim}{\mathrm{H}}=i \omega \varepsilon_{0} \underset{\sim}{\mathrm{K}} \cdot \underset{\sim}{\mathrm{E}}
\end{aligned}
$$


where $\underset{\approx}{\mathrm{K}}$ is the plasma dielectric tensor of the cavity medium, which can depend upon position.

For a lossless medium, the wave equations can be solved by writing the driven fields $\underset{\sim}{\mathrm{H}}$ as a sum over all eigenmodes of the system such that

$$
\underset{\sim}{\mathrm{H}}=\sum_{\mathrm{q}} \mathrm{D}_{\mathrm{q}} \underset{\sim \mathrm{G}}{\mathrm{H}}
$$

where the eigenmodes are solutions to the equations

$$
\begin{aligned}
& -\nabla \times \underset{\sim q}{\mathrm{E}}=i \omega_{\mathrm{q}}{ }^{\mu} \stackrel{\sim}{\mathrm{H}}_{\mathrm{g}} \\
& \nabla \times \underset{\sim q}{\dot{\dot{H}}}=i \omega_{g} \varepsilon_{0} \underset{\approx q}{\mathrm{~K}} \cdot E_{\sim q}^{E} .
\end{aligned}
$$

Both the driven fields and the eigenmodes must safisfy the boundary conditions that the tangential components of the electric field are zero on the surface of the cavity. Using the orthogonality relations between eigenmodes and the boundary conditions it can be shown that the dominant terms in Eq. (4) are those for which $\omega_{q} \approx \omega$. Specifically, if the driving frequency equals one eigenfreguency, say

$$
\omega=\omega_{g},
$$

-. the amplitude of the resonant term become infinite and the $\mathrm{g}^{\prime}$ th term is the dominant term of Eg. (4).

When small dielectric losses are to be included, the resonant term is still dominant and its amplitude is determined by the dissipation. The wave equations for the undriven cavity are 


$$
\begin{aligned}
& -\nabla \times \underset{\sim q^{\prime}}{E}=\eta_{g} \mu_{0} \underset{\sim q}{\mathrm{H}_{\mathrm{q}}^{\prime}} \\
& \nabla \times \underset{\sim \mathrm{q}^{\prime}}{\mathrm{H}}=\eta_{\mathrm{g}} \varepsilon_{\mathrm{O}} \underset{\approx}{\mathrm{K}(\eta)} \cdot \underline{\sim}_{\mathrm{q}^{\prime}} .
\end{aligned}
$$

The small perturbation in the dielectric tensor due to losses causes the time dependence of the mode of oscillation to change from $e^{-i \omega_{g} t}$ to $e^{-n_{g} t}$, where $n_{g}$ is complex.

A pcrturbation expansion of Eq. (6) abnut the pigenfunctions (5), shown by Auer, Hurwits \& Milior (1958) and stix (19̧\%), yields that

$$
\left|\delta n_{q}\right|=\left|n_{g}-i \omega_{q}\right| \approx \frac{\omega_{g}}{2 Q_{g}}
$$

where $Q_{q}$ is the quality factor of the $q^{\prime}$ th mode in the cavity, given by

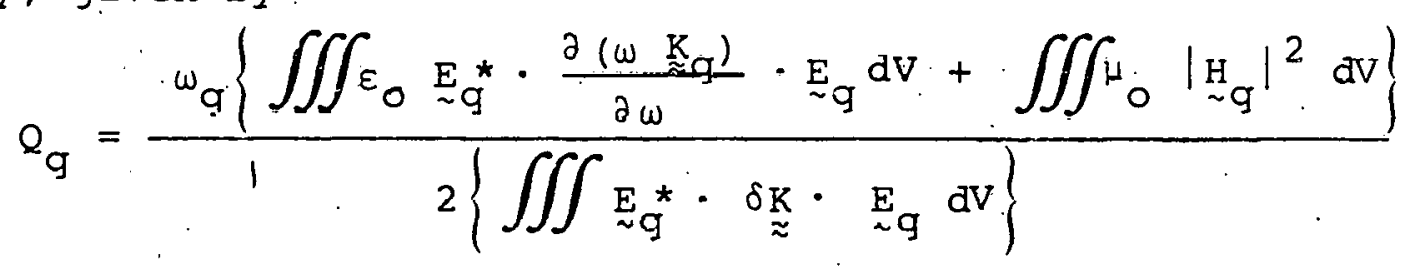

and $\delta \underset{\approx}{K}$ is the anti-Hermitian part of the dielectrictensor which produces energy dissipation.

Approximate solutions for the driven cavity in the resonant situation can be obtained by writing

$$
\underset{\sim}{\mathrm{H}} \approx \mathrm{D}_{\mathrm{q}} \underset{\sim \mathrm{G}}{\mathrm{H}} \mathrm{q}^{\prime}
$$

Since the drive frequency is resonant with the $g^{\prime}$ th mode we have

$$
\underset{\sim}{E} \approx D_{q} \underset{\sim q}{ }{ }^{\prime}
$$


and thus Eqs. (3) become

$$
\begin{aligned}
& -D_{q} \nabla \times \underset{\sim q}{E} q^{\prime}=i \omega_{g} \mu_{0} D_{q} \underset{\sim q}{H} q^{\prime}+\underset{\sim s}{M}
\end{aligned}
$$

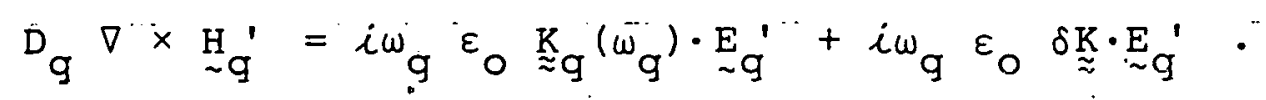

Equations (8) and (5) can be combined and integrated over the volume of the cavity to give

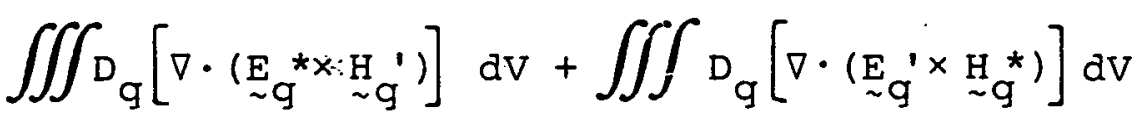

$$
\begin{aligned}
& =i \omega_{\mathrm{q}} \varepsilon_{\mathrm{O}} \cdot \mathrm{D}_{\mathrm{q}} \iiint_{\sim \mathrm{q}^{\mathrm{E}}}^{*} \cdot \delta \underset{\approx}{\mathrm{K}} \cdot{\underset{\sim \mathrm{q}}{\mathrm{E}}}^{\prime} \mathrm{dV}-\iiint_{\sim_{\mathrm{q}}^{\mathrm{H}}}^{*} \cdot \underset{\sim \mathrm{S}}{\mathrm{M}_{\mathrm{S}}} \mathrm{dV}
\end{aligned}
$$

Both expressions on the left hand side can be reduced to surface integrals, and since both $\underset{\sim q}{E}$ and $\underset{\sim q}{E}$ ' satisfy boundary conditions on the surface, we obtain in the limit of small losses

$$
\left|D_{\mathrm{q}}\right|=\frac{\iiint_{\sim \mathrm{G}}^{\mathrm{H}} \cdot \underset{\sim}{\mathrm{M}} \mathrm{S} d \underline{\mathrm{dV}}}{i \omega_{\mathrm{q}} \varepsilon_{\mathrm{O}} \iiint_{\sim \mathrm{q}^{*}}^{\mathrm{E}^{*}} \cdot \delta \underset{\sim}{\mathrm{K}} \cdot \underset{\sim \mathrm{q}}{\mathrm{E}} \mathrm{dV}}
$$

Using equation (7), the above expression can be rewritten

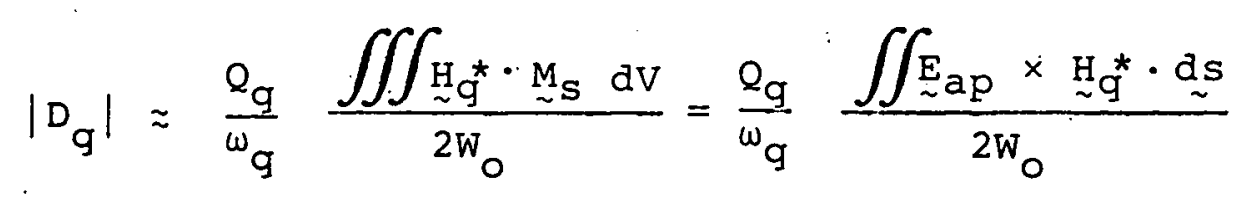

where $w_{0}$ is the time averaged total eigenmode energy in the cavity given by

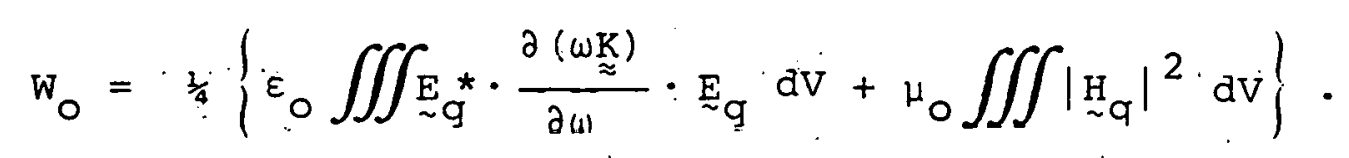




\section{Effective Aperture Impedance}

We now take the component of $\underset{\sim q}{\mathrm{H}}$ which is perpendicular to both $\underset{\sim a p}{E}$ and $\underset{\sim}{\hat{n}}$ to be real. The conjugate complex power at the aperture is thus

$$
\mathrm{P}_{\mathrm{C}^{*}}^{*}=\iint_{\text {aperture }}(\underset{\sim \mathrm{ap}}{\mathrm{E}} \times \underset{\sim}{\mathrm{H}}) \cdot \mathrm{ds} \approx \frac{\mathrm{Q}_{\mathrm{q}}}{2 \omega_{\mathrm{q}} \mathrm{W}_{\mathrm{O}}}\left[\iint\left(\underset{\sim \mathrm{ap}}{\mathrm{E}} \times \underset{\sim q}{\mathrm{H}_{\mathrm{q}}}\right) \cdot \mathrm{ds}_{\sim}\right]^{2} .
$$

Equating the complex aperture power calculated from within the waveguide to the complex power computed from within the cavity, we obtain

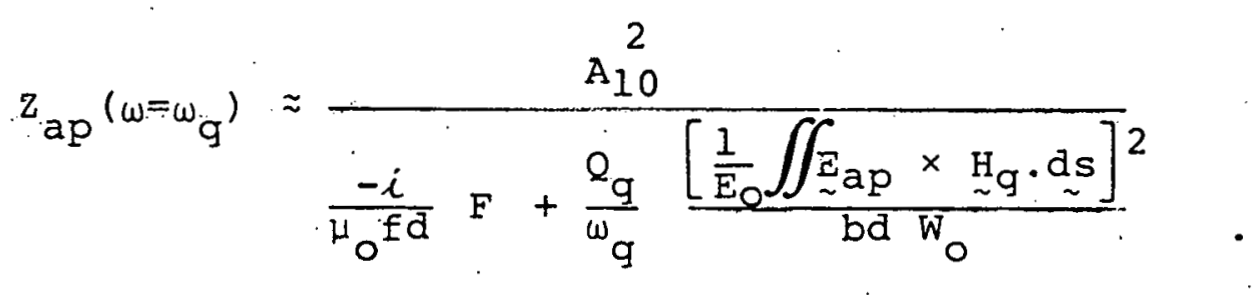

The dimsionless quantity $\dot{F}$, given by

$$
F=\frac{i \mu_{o} f d}{2} \sum_{E=2}^{\infty} \frac{A_{p o}^{2}}{Z_{j u}}
$$

is related to the contribution to the effective aperture impedance of the cut-off modes which are generated because the aperture width $d^{\prime}$ is less than the waveguide width $d$. These cut-off modes store energy in such a way that their contribution to $\underline{Z}_{\text {ap }}$ is inductive. $F$ is plotted as a function of (d'/d) for several values of the parameter $\left(d / \lambda_{0}\right)$ in Fig. 3 . 
When the aperture is maximum $\left(d^{\prime}=d\right)$

$$
\mathrm{z}_{\mathrm{ap}}\left(\omega=\omega_{\mathrm{q}}\right) \approx \mathrm{db}\left(\frac{\omega_{\mathrm{q}}}{\mathrm{Q}_{\mathrm{q}}}\right) \frac{\mathrm{w}_{\mathrm{O}}}{\left[\frac{1}{\mathrm{E}_{\mathrm{O}}} \iiint_{\sim \mathrm{ap}} \times \underset{\sim q}{\mathrm{H}_{\mathrm{q}}} \cdot \mathrm{ds}_{\sim}\right]^{2}} \cdot
$$

The impedance is real and thus represents a resistive load. Contributions from non-resonant cavity modes (which have been neglected in this model) would add a reactive component to $\mathrm{Z}_{\text {ap' }}$ ' but if $Q_{q}$ is large enough, this added component is small. Note that if $\mathrm{Q}_{\mathrm{q}} \rightarrow \infty, \mathrm{Z}_{\mathrm{ap}} \rightarrow 0$ and the aperture behaves as if it were closed by an infinitely conducting barrier (Morse \& Feshbach (1953)).

\section{LOOP COUPLING MODEL}

Coupling to cavity eigenmodes can also be achieved by an electric current source term $J_{S}$ within the cavity. The wave equations are

$$
\begin{aligned}
& -\nabla \times \underset{\sim}{E}=i \omega \mu_{0} \underset{\sim}{H} \\
& \nabla \times \underset{\sim}{H}=i \omega \varepsilon_{0} \underset{\sim}{\mathrm{K}} \cdot \underset{\sim}{E}+{\underset{\sim}{S}}_{S}
\end{aligned}
$$

and can be solved in the resonant approximation $\left(\omega=\omega_{q}\right)$ by an analogous method to that described in the previous section. The amplitude of the resonant term is

$$
D_{g} \approx\left(\frac{Q_{g}}{\omega_{g}}\right) \frac{\iiint_{\sim} J_{s} \cdot E_{g}^{*} \cdot d V}{2 W_{o}}
$$

The input impedance of the loop is defined as

$$
Z_{L}=\frac{I}{I_{0}^{2}} \iiint_{\sim} \underset{\sim}{E} \cdot J_{S} d V
$$


where $I_{0}$ is the input current to the loop, and using the resonant mode expansion. for the driven cavity fields, we obtain

$$
\mathrm{z}_{L}\left(\omega=\omega_{\mathrm{q}}\right)=\left(\frac{\dot{Q}_{\mathrm{q}}}{\omega_{\mathrm{g}}}\right) \frac{\left[\frac{\mathrm{I}}{\mathrm{I}_{\mathrm{O}}} \iiint \underset{\sim}{\mathrm{J} \cdot \mathrm{F}_{\mathrm{g}}} \mathrm{dV}\right]^{2}}{2 \mathrm{w}_{\mathrm{O}}} .
$$

We assume that part of the loop is placed within the cavity, the return path being along the cavity wall where the tangential component of $\mathrm{E}$ is zero. Also, if the loop is short and narrow,

$$
\frac{I}{I_{0}} \iiint J \cdot E_{\cdot q} d V=\int_{100 p} E_{q} \cdot d l=\omega \phi
$$

where $\phi$ is the eigenmode magnetic flux enclosed by the loop. Consequently

or

$$
\begin{aligned}
Z_{L}\left(\omega=\omega_{q}\right) & =\frac{Q \omega_{g} \phi^{2}}{2 W_{O}} \\
\frac{\omega_{g} W_{O}}{Q} & =\frac{\omega_{g}^{2} \phi^{2}}{2 Z_{L}} \quad \therefore
\end{aligned}
$$

Thus the time averaged work done by the back emf of the loop equals the energy lost within the cavity. Equation (ii) is the basis for calculations of loop coupling to eigenmodes described by Paoloni (1975a).

\section{PLASMA MODEL}

We take the plasma to be a uniform column of radius $p$, which is surrounded by a vacuum annulus from $p$ to the surface to the metal chamber of radius a. The $z_{c}$ axis is centered 
on the cylinder axis and the $\theta=0$ axis runs through either the center of the waveguide aperture; as shown in Fig. 4 , or the center of the coupling loop.

Perkins (1972) and Swanson (1974) have shown that the wave functions of the fast wave in toroidal geometry are essentially the same as those in cylindrical geometry provided the aspect ratio is moderately large. The axial (toroidal) magnetic field of the fast eigenmode at $r=a$, is given approximately by

$$
\begin{aligned}
B_{z}(r=a) \approx & B_{z 0} e^{-k_{n}(a-p)} J_{m}\left(k_{\perp} a\right) \text { cos }\left(k_{n} z_{c}\right) e^{-j(\omega t-m \theta)} \\
\text { where } m= & \text { the azimuthal mode number } \\
k_{n}= & \frac{2 \pi N}{l_{t}} \text { is the parallel. wave number which is related } \\
& \text { to the perpendicular wave number } k_{\perp} \text { by the } \\
& \text { dispersion relation for fast waves } \\
l_{t}= & \text { circumference of the toroidal cavity }=2 \pi \mathrm{R} \\
\mathrm{R}= & \text { major radius } \\
\mathrm{N}= & \text { number of wavelengths } \lambda_{n} \text { around the torus. }
\end{aligned}
$$

Provided $k_{\perp}<\omega / V_{A}$ (where $V_{A}$ is the Alfvén velocity) the perpendicular wave number is determined approximately by the equation

$$
J_{m \cdots 1}(k, a)=0
$$

Also, ignoring the lefthand circularly polarized component of the fast wave and the energy stored in the vacuum layer, the time averaged total energy of the standing eigenwave in the torus is approximately 
$W_{0}=\frac{B_{z O}^{2}}{8 \mu_{0}} J_{m}^{2}\left(k_{\perp} a\right)\left[\frac{1}{2 k_{\perp}^{2}}\left(\frac{k_{A}^{2}}{(1+\Omega)^{2}}+k_{11}{ }^{2}\right)\left(\frac{C}{k_{n} A}+1\right)^{2}+1\right]\left(\pi a^{2} l_{T}\right)$

where $\frac{C}{k_{1} A}=\frac{\left(k_{n}^{2}+k_{\perp}^{2}\right)\left(\frac{\omega}{\omega l}\right)}{\left(\frac{\omega^{2}}{v_{A}^{2}}-k_{11}^{2}\right)}$

is related to the wave polarization and is approximately unity Eor fast waver.

The quantity in square brackets represents the ratio of the total wave energy to the energy in the axial magnetic field component.

The above equations for the MHD waves were derived by ignoring the electron mass term in the cold plasma dielectric tensor. An accurate derivation of the wave fields in the vacuum layer outside the plasma column reguires retention of these terms as shown by Allis, Buchsbaum and Bers (1963), Hosea and Sinclair (1969), and Paotoni (1975b), among others. ilhe method can readily be applied to the above calculations but the boundary conditions and wave fields reguire numerical solution.

\section{COUPLING IMPEDANCE}

1. Waveguide Coupling

We assume that the aperture height $b$ is much less than the minor radius $a$, so that the aperture field can be written in cavity coordinates

$$
\underset{\sim a p}{E_{0}} \approx E_{0} \cos \frac{\pi z}{d^{\prime}} \underline{\theta}
$$


The reguired integral in the impedance formula is

$$
\iint_{\text {aperture }}\left(\underset{\sim a p}{E}{ }_{\sim q}^{H}\right) \cdot d s=\iint_{\text {aperture }} E_{a p} H_{z_{C}}(r=a) a d z_{C} d \theta
$$

$\approx a \frac{B_{z O}}{\mu_{0}} E_{0} J_{m}\left(k_{\perp} a\right) e^{-k_{n}(a-p)}\left[\frac{2}{m} \sin \frac{m b}{2 a}\right]\left[\frac{\frac{2 \pi}{d^{\prime}} \cos \left(\frac{d^{\prime} k_{n}}{2}\right)}{\left(\left(\frac{\pi}{d^{\top}}\right)^{2}-k_{n}^{2}\right)}\right]$

The first quantity in brackets on the right hand side represents the Fourier amplitude of the aperture field in the $\theta$ plane, while the second term is the Fourier amplitude in the $\mathrm{z}_{\mathrm{C}}$ plane.

Considering only the simple case when the aperture area equals the waveguide cross-sectional area: $\left(d^{\prime}=d\right)$, we obtain

$$
z_{a p}=\frac{\pi^{3}}{128} \mu_{0} \ell_{t}\left(\frac{\omega}{Q}\right)\left(\frac{b}{d}\right) \frac{\left[\frac{1}{2 k_{\perp}^{2}}\left(\frac{k_{A}{ }^{2}}{(1+\Omega)^{2}}+k_{n}{ }^{2}\right)\left(\frac{c}{k_{n} A}+1\right)^{2}+1\right]}{\left[e^{-k_{n}(a-p)}\left(\frac{1}{m} \sin \frac{m b}{2 a}\right) \cdot \frac{\cos \frac{k_{n} d}{2}}{\left[1-\left(\frac{k_{n} a}{\pi}\right)^{2}\right]}\right]^{2}} .
$$

2. Loop Coupling

We consider an infinitesmally thin loop of length $b$, oriented in the poloidal direction on the outside region of the torus. The loop is a distance $\Delta$ from the chamber surface and $\delta=(a-p)>\Delta$ is the thickness of the vacuum layer. The flux enclosed by the loop is approximately

$$
\phi \approx B_{z O} J_{m}\left(k_{\perp} a\right)\left(a \Delta e^{-k_{n}\left(\delta-\frac{\Delta}{2}\right)}\right)\left(\frac{2}{m} \sin \frac{m b}{2 a}\right)
$$


and substituting into the coupling impedance formula (11) we obtain

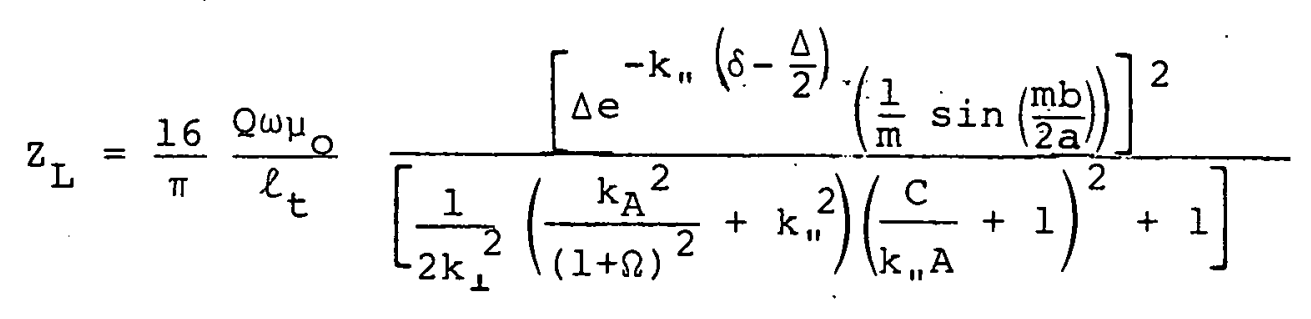

CONDITIONS FOR OPTIMAL COUPLING

The: RF source is assumed to be matched to the transmission line that delivers energy from the source to the coupler. Maximum power can thus be transferred to the plasma when the coupling impedance equals the characteristic impedance of the transmission system. If this condition is not met then matching devices, such as tuning stubs, are required in the transmission line to minimize the power reflection from the load.

Ideally, one would wish to choose an eigenmode for which the effective loading impedance of the coupler equals that of the transmission system. However, such conditions would be fortuitous and matching stubs will probably be reguired. It is thus necessary to select eigenmodes which are most strongly coupled to the launcher and then use the external matching network to optimize the power transfer. For a waveguide couplcr it is necessary to select modes for which the aperture impedance is minimal while for loop couplers, the impedance should be maximal. The coupling can be degraded (or improved) by increasing (or decreasing) the distance from the coupler to the plasma surface. 
An interesting result which can be obtained by multiplying Egs. (12) and (13) is that, for long wavelengths, the product $\left(Z_{\operatorname{ap}} \times z_{L}\right)$ is independent of $Q$ and the eigenmode within the cavity and depends only on geometrical quantities. The result suggests that once the loading impedance of one coupler is known, the other can be inferred from the geometrical configuration.

The final condition reguired to maintain efficient energy transfer is that the cavity remains in resonance for extended times during which the plasma parameters may vary. The eigenfrequencies of the cavity will be time dependent and thus a feedback scheme will be necessary to maintain a resonant condition. Consequently the external coupling scheme must be sufficiently broadband to allow frequency shifts to follow the resonance.

\section{EXAMPLE}

As an example we calculate loop and waveguide coupling to fast toroidal eigenmodes in PLT at a frequency of $60 \mathrm{MHz}$.

\section{Eighth. Turn Loop}

We consider the coupling impedance of a single eighth turn loop $(b \approx 40 \mathrm{~cm})$ which is placed $5 \mathrm{~cm}$ from the vacuum vessel wall. The $Q^{\prime}$ s of the cavity for cyclotron harmonic $(\Omega=2$ in center plasma) and electron transit-time damping, $\mathbb{Q}_{\mathrm{CH}}$ and $\mathrm{Q}_{\mathrm{ETT}}$ respectively, have been calculated previously by paoloni (1975a) for the conditions:

$$
\begin{array}{ll}
\text { plasma radius } & \mathrm{p}=45 \mathrm{~cm} \\
\text { minor radius } & \mathrm{a}=50 \mathrm{~cm} \\
\text { major radius } & \mathrm{R}=130 \mathrm{~cm}
\end{array}
$$




$$
\begin{array}{ll}
\text { electron density } \mathrm{n}_{\mathrm{e}}=5 \times 10^{13} / \mathrm{cm}^{3} \\
\text { electron temp } & \mathrm{T}_{\mathrm{e}}=1 \mathrm{keV} \\
\text { ion temp } & \mathrm{T}_{i}=1 \mathrm{keV}
\end{array}
$$

Some approximate wave parameters and the coupling impedances for various radial orders ( $n$ ) of $m=0$ fast modes are as follows (in MKS units):

\begin{tabular}{rrrrrrrrr}
$\mathrm{n}$ & $\mathrm{k}_{\perp}$ & \multicolumn{1}{c}{$\mathrm{k}_{n}$} & $\lambda_{n}$ & $\mathrm{~N}$ & $\frac{\mathrm{C}}{\mathrm{k}_{n} \mathrm{~A}}$ & $Q_{\mathrm{CH}}$ & $\mathrm{Q}_{\mathrm{ETT}}$ & $\mathrm{z}_{\mathrm{L}} / \mathrm{Q}(\mathrm{ohm})$ \\
1 & 7.66 & 24.7 & .26 & 31 & 1.04 & $>10^{7}$ & 3865 & $3 \times 10^{-4}$ \\
2 & 14 & 23.4 & .28 & 29 & 1.08 & $8 \times 10^{5}$ & 1250 & $2 \times 10^{-3}$ \\
3 & 20.4 & 21.2 & .31 & 26 & 1.16 & $8 \times 10^{4}$ & 673 & $2.7 \times 10^{-3}$ \\
4 & 26.6 & 18.4 & .37 & 22 & 1.27 & $2 \times 10^{4}$ & 508 & $6 \times 10^{-3}$ \\
5 & 33 & 14.4 & .48 & 17 & 1.48 & 4500 & 605 & $1.1 \times 10^{-2}$ \\
6 & 39.2 & 9.2 & .74 & 11 & 1.80 & 1570 & 4840 & $2.3 \times 10^{-2}$
\end{tabular}

For the longest wavelength $(\mathrm{N}=1)$

$$
\mathrm{Z}_{\mathrm{L}} \approx 0.05 \mathrm{Q}
$$

The results suggest that the coupling impedance is smail (less than $1 \Omega$ ) for short wavelength modes but increases to more than $20 \Omega$. for the longer wavelengths.

2. Waveguide

We consider the possiblitty of using a loaded waveguide in PLT. Since the operating frequency is $60 \mathrm{MHz}$ and the guide must fit into a large port of the vacuum vessel, we find that a dielectric constant of about 80 is required. Two suitable

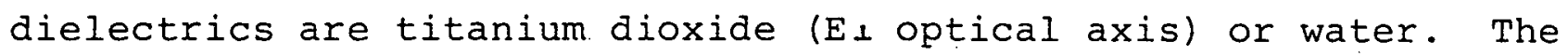
properties given by Von Hippel (1954) for $\mathrm{TiO}_{2}$ are: 
$\begin{array}{cccccc}\mathrm{T}^{\circ} \mathrm{C} & & \frac{1 \mathrm{MHz}}{25^{\circ} \mathrm{C}} & \frac{10 \mathrm{MHz}}{85.8} & \frac{100 \mathrm{MHz}}{-} & 300 \mathrm{MHz} \\ & \begin{array}{llll}\varepsilon_{\mathrm{r}} / \varepsilon_{0} \\ \tan \delta\end{array} & \begin{array}{c}85.8 \\ 2 \times 10^{-4}\end{array} & 2 \times 10^{-4} & - & -\end{array}$

and for pure de-ionized water:

$\begin{array}{rrrrrr}1.5^{\circ} \mathrm{C} & \varepsilon_{r} / \varepsilon_{0} & 87 & 87 & 87 & 87 \\ & \tan \delta & 190 \times 10^{-4} & 20 \times 10^{-4} & 70 \times 10^{-4} & 320 \times 10^{-4} \\ 25^{\circ} \mathrm{C} & \varepsilon_{r} / \varepsilon_{0} & 78.2 & 78.2 & 78 & 77.5 \\ & \tan \delta & 400 \times 10^{-4} & 46 \times 10^{-4} & 50 \times 10^{-4} & 160 \times 10^{-4}\end{array}$

Some relevant waveguide formulas that are useful are:

$$
\begin{array}{ll}
f_{10}=1 /(2 \mathrm{~d} \sqrt{\mu \varepsilon}) & { }^{T E_{10}} \text { cutoff frequency } \\
\lambda_{g}=\lambda_{0}\left(1-\left(f_{10} / f\right)^{2}\right)^{-1 / 2} & { }^{T E_{10}} \text { waveguide wavelength } \\
Z_{10}=n \lambda_{g} / \lambda_{0} & { }^{T E_{10}} \text { waveguide impedance } \\
L=I_{0} \lambda_{0} / \lambda_{g} & { }^{T E_{10}} \text { attenuation length due } \\
& \text { to dielectric losses } \\
I_{0}=\lambda_{0} /(\pi \tan \delta) & \text { attentuation length in } \\
& \text { waveguide medium } \\
\tan \delta & \text { loss tangent of waveguide } \\
& \text { medium. }
\end{array}
$$

Operating at $60 \mathrm{MHz}$ with

$$
f_{10}=0.8 \mathrm{f}
$$

and

$$
\frac{b}{d}=\frac{1}{2}
$$


we obtain

$$
\begin{aligned}
& \mathrm{d} \approx 35 \mathrm{~cm} \\
& \lambda_{\mathrm{g}} \approx 94 \mathrm{~cm} \\
& \mathrm{~L} \approx 22 \text { meters (water } 25^{\circ} \mathrm{C} \text { ) } \\
& \mathrm{L} \approx 540 \text { meters }\left(\mathrm{TiO}_{2}\right) \\
& \mathrm{z}_{10} \approx 71 \Omega
\end{aligned}
$$

The peak electric field for a power flow of 1 MW in the matched guide is approximately $7 \times 10^{4} \mathrm{v} / \mathrm{m}$.

\begin{tabular}{|c|c|c|c|c|}
\hline$n$ & $N$ & $\cdot{ }^{Q} \mathrm{CH}$ & $Q_{\text {ETT }}$ & $Q \times z_{a p}(o h m)$ \\
\hline 1 & 31 & $>10^{7}$ & 3865 & $2 \times 10^{9}$ \\
\hline 2 & $2 \overline{9}$ & $8 \times 10^{5}$ & 1250 & $2 \times 10^{8}$ \\
\hline 3 & 26 & $8 \times 10^{4}$ & 673 & $2.2 \times 10^{7}$ \\
\hline 4 & 22 & $2 \times 10^{4}$ & 508 & $3.6 \times 10^{6}$ \\
\hline 5 & 17 & 4500 & 605 & $7 \times 10^{5}$ \\
\hline 6 & 11 & 1570 & 4840 & $1.5 \times 10^{5}$ \\
\hline
\end{tabular}

We assume the same plasma conditions as for loop coupling. and take the distance from the waveguide aperture to the plasma to be $5 \mathrm{~cm}$. The effective loading impedances for various $\mathrm{m}=0$ modes are

For the longest wavelength $(\mathrm{N}=1)$

$$
z_{\text {ap }} \approx 4 \times 10^{4} / \mathrm{Q} \text { ohms. }
$$

Since the characteristic impedance of the loaded guide is $71 \Omega$, the $Q$ required to match the waveguide to long wavelength eigenmodes lies in the range

$$
500 \leqslant Q \leqslant 1000
$$


Given the great variety of modes in PLT, each with its own $Q$, it is possible to select one or more that will strongly couple to the waveguide. "Furthermore, the loading can be altered by changing the distance from the aperture to the plasma so that for strongly coupled"modes the impedance can be selected to nearly match the guide.

A conceptual design of a titanium dioxide or water filled waveguide for PLT is given in Fig. 5. The coax/waveguide transition would consist of a "doorknob transformer" which is capable of handing high powers without breakdown. The transition is broadband and should provide less than 5\% reflection over a $10 \%$ range about the center frequency, thus enabling frequency changes to follow the eigenmode as the plasma conditions vary with time.

The maximum length of the waveguide is determined by the maximum permissible power loss in the dielectric medium. If the length of the guide is equal to half a waveguide wavelength, there is less than 58 power loss per pass for a water filled guide and insigrificant loss for à 'lio 2 filled guide. The minimum length is determined by the need to have higher order waveguide modes negligible. For an aspect ratio of $b / d=1 / 2$, the next higher modes are the $\mathrm{TE}_{01}$ and $\mathrm{TE}_{20}$ modes which have the same cut-off frequency. The attenuation length of these cut-off modes is about $6 \mathrm{cms}$.

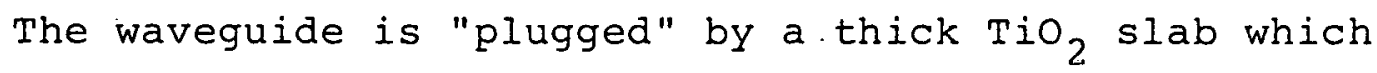
provides the vacuum. seal to the plasma chamber. Two layers of 
metal strips at the waveguide mouth are placed perpendicular to the waveguide electric field as shown in Fig. 6. The layers overlap so that the ceramic is effectively shielded from the plasma. The region between the strips is partially filled with material of high dielectric constant (e.g. Tam Ticon $\varepsilon_{r}=167$ ) to keep that region of the waveguide above cut-off. Estimates of the power reflection from such a Faraday screen have been obtained from bench tests. Scaled down Faraday screens were constructed for an air filled WR 229 (2.29" $\times 1.14^{\prime \prime)}$ waveguide operating at $3.4 \mathrm{GHz}$. One screen consisted of a single layer of five evenly spaced copper strips $1 / 8 "$ wide and $0.025 "$ thick. The other consisted of two overlapping layers spaced $3 / 16$ " apart. The dimensions, spacing and wavelength are scaled roughly as envisaged in Fig. 6. When placed in the middle of a long section of waveguide, the single screen caused $0.6 \%$ power reflection while the double screen produced $1 \%$ reflection. The results suggest that the presence of a Faraday screen on the loaded waveguide should cause only very small additional reflection in the guide.

\section{CONCLUSIUN}

The loading impedancce fur loops and wavcguide are related in such a way that when one impedance is known, the other can be inferred from the geometrical configuration. Approximate calculations for PLT suggest that the loading of either loops or waveguides can be sufficient to allow efficient energy transfer to the cavity. Coupling is best for the longer wavelengths since 
the eigenmode fields are larger near the antenna. The results indicate that under certain conditions it may be possible to match the antennas to the cavity but usually an external matching circuit will be required.

\section{ACKNOWLEDGEMENTS}

The author wishes to thank.W. M. Hooke, J. C. Hosea, and T. H. Stix for useful discussion during the preparation of this report.

This work was supported. by the U. S. Energy Research and Development Administration Contract AT (11-1)-3073. 
REFERENCES

J. Adam, M. Chance, H. Eubank, W. Getty, E. Hinnov, W. Hooke, J. Hosea, F. Jobes, F. Perkins; R: Sinclair, J. Sperling, and H. Takahashi, Princeton Plasma Physics Laboratory Matt 1094 (1974). W. P. Allis, S. J. Buchsbaum and A. Bers, Waves in Anistropic Plasmas, MIT Press, Cambridge, Mass. (1963).

P. L. Auer, H. Hurwitz, and R. D. Miller, General Electric Research Laboratory Report \# 58-RL-2020 (1958).

R. F. Harrington, Time Harmonic Electromagnetic Fields, McGrawHill Book Co., N.Y. (1961).

J. C. Hosea and R. M. Sinclair, Phys. Rev: Lett. 23, 3 (1969).

J. C. Hosea and R. M. Sinclair, Phys. Fluids 13, 701 (1970).

J. C. Hosea and W. M. Hooke, Princeton Plasma Physics Laboratory Matt 940 (1972).

J. C. Hosea and W. M. Hooke, Phys. Rev. Lett. 31, 150 (1973).

P. M. Morse and H. Feshbach, Methods of Theoretical Physics, Vol. II, MCGraw-HiIl Book Co., N.Y. (1953).

F.J. Paoloni, Princeton Plasma Physics Laboratory Matt 1119 , (1975 a).

F. J. Paoloni, Phys. Fluids 18, 640 (1975 b). 
F. W. Perkins, International School of Plasma. Physics, "Symposium on Plasma. Heating and Injection," Varenna Italy (1973). T. H. Stix, The Theory of Plasma Waves, McGraw-Hill Book Co., N.Y. (1962).

T. H. Stix, Princeton Plasma Physics Laboratory Matt 1113 (1975)

- to be published in Nuclear Fusion.

D. G. Swanson, Phys. Fluids 17,2241 (1974).

A. R. Von Hippel, Dielectric Materials and Applications, MIT Press (1954). 


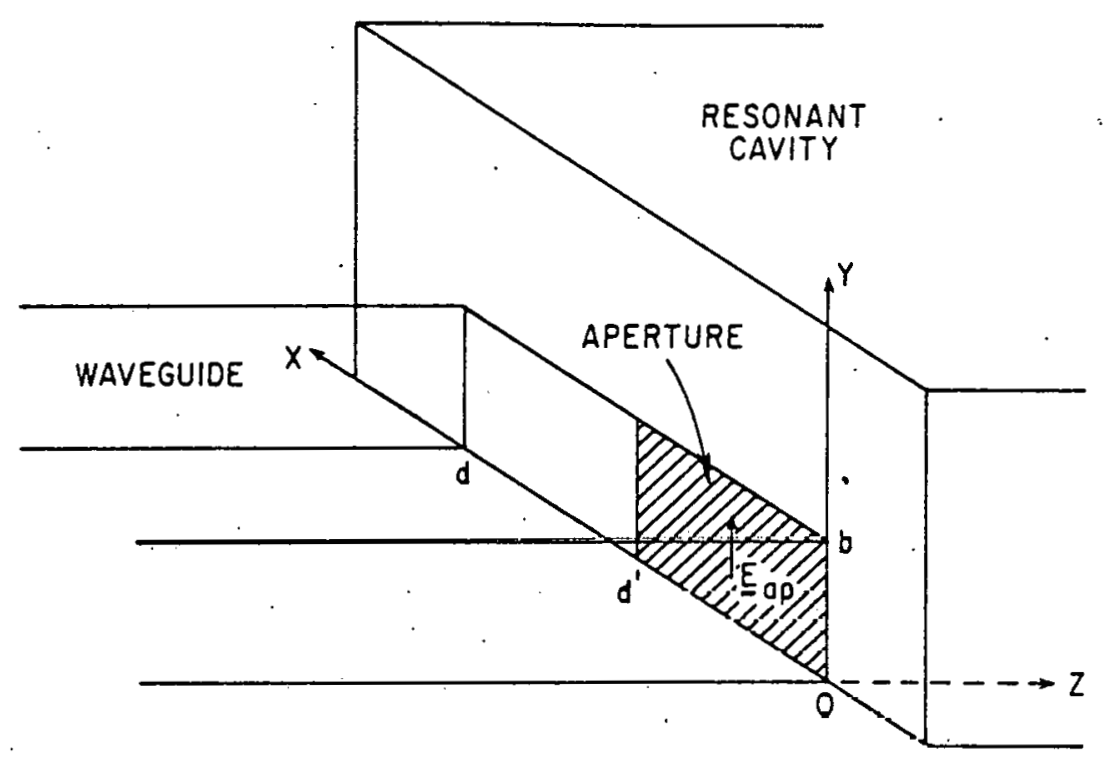

753916

Fig. 1. Schematic diagram of the waveguide aperture and waveguide coordinates.
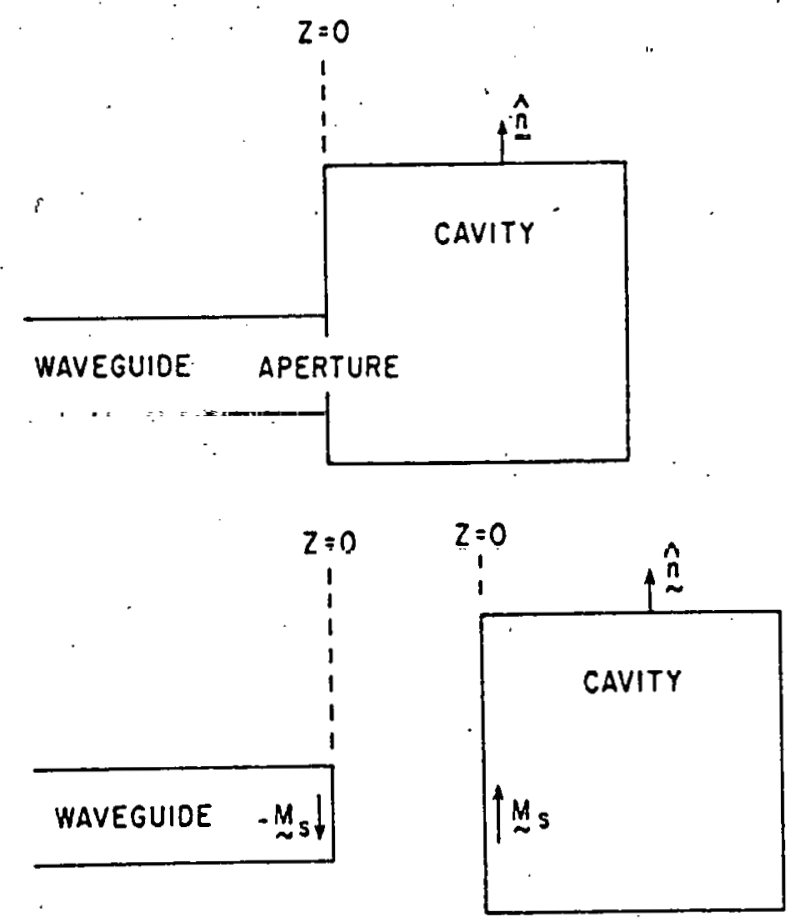

753913

Fig. 2. A sketch demonstrating the division of the waveguide from the cavity by the use of a conceptual magnetic source ${\underset{\sim}{M}}_{S}$. 


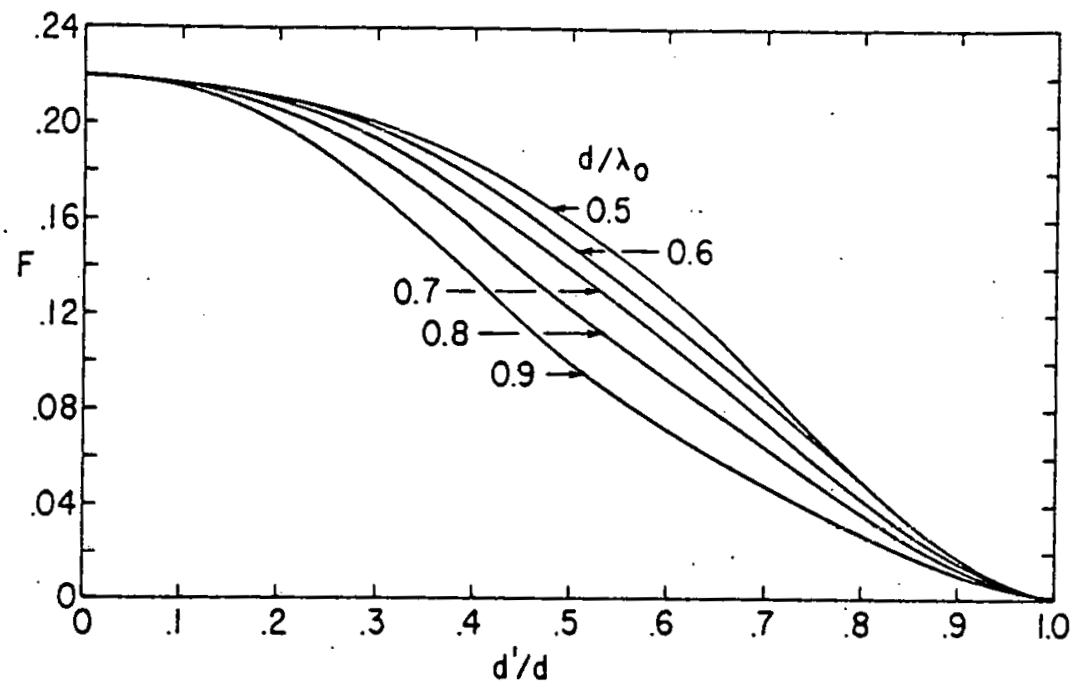

753915

Fig. 3. The dimensionless quantity $F$ as a function of $d / d$ for different values of the parameter $d / \lambda_{0}$.

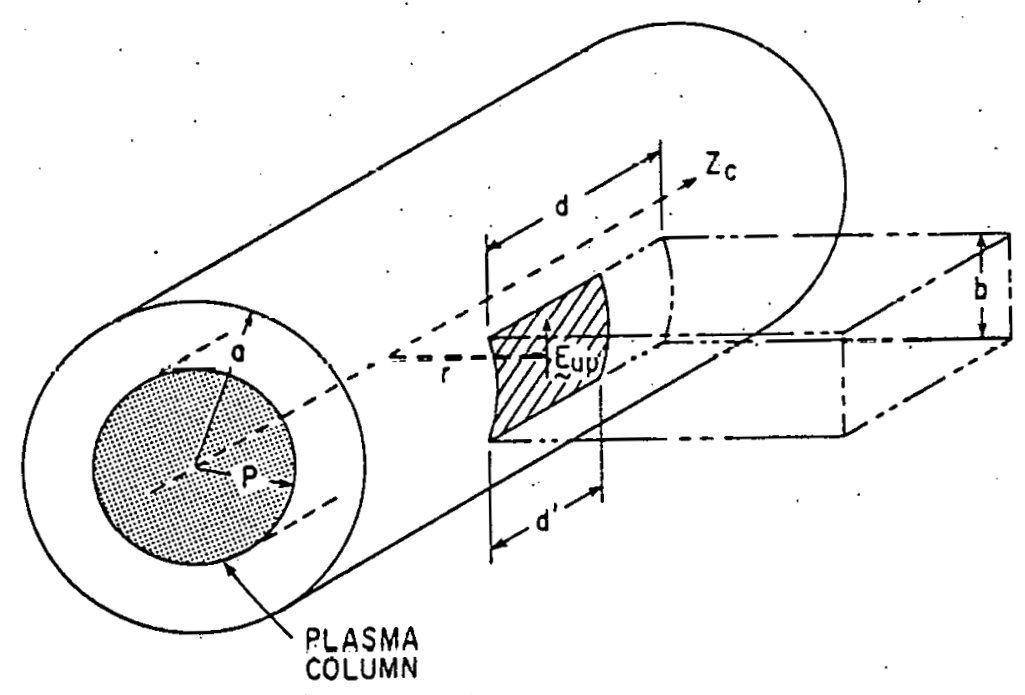

753914

Fig. 4. Diagram showing the cylindrical cavity coordinate system. The radii of the chamber and the plasma are a and $p$, respectively. The waveguide is mounted perpendicular to the chamber wall and the $\theta=0$ axis runs through the center of the aperture. 


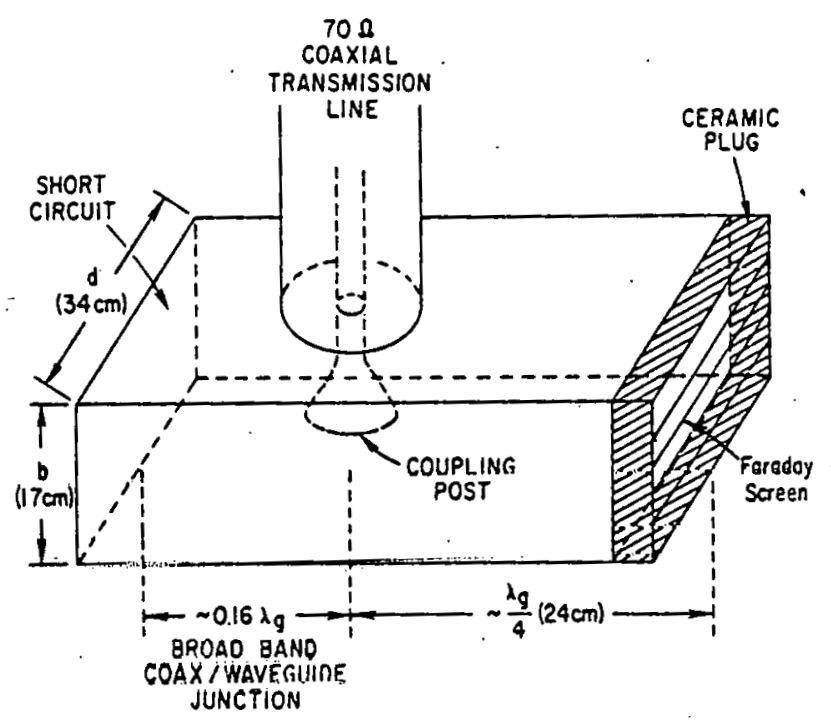

75607.5

Fig. 5. A conceptual design of a $\mathrm{TiO}_{2}$ or water filled waveguide for PIT.

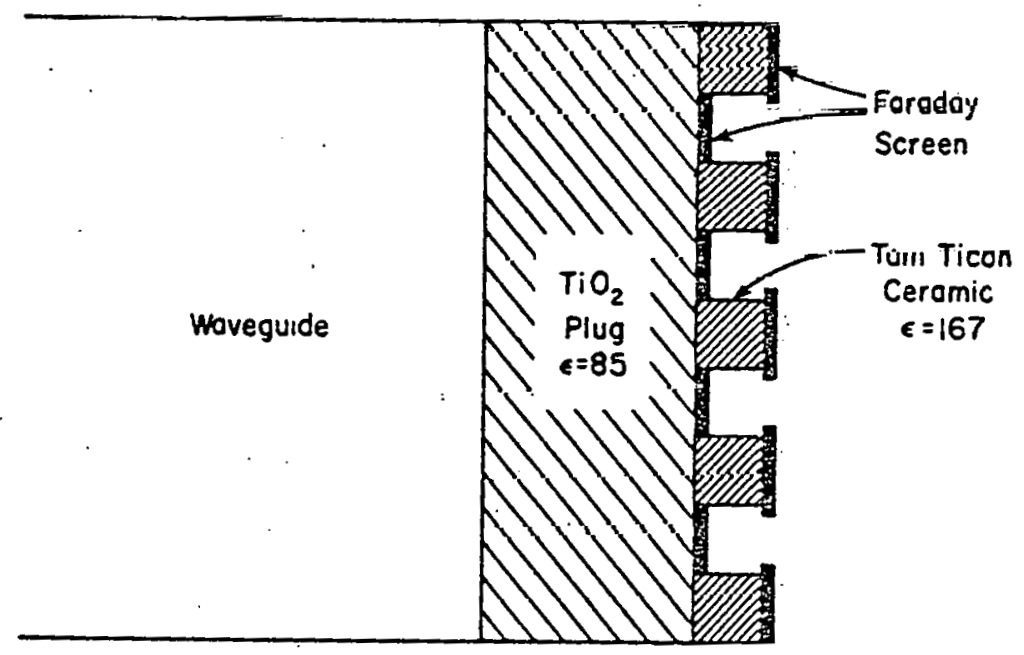

755074

Fig. 6. The Faraday screen placed on the mouth of the waveguide facing the plasma. 